\section{Is it language that makes humans intelligent?}

\author{
Jo Van Herwegen and Annette Karmiloff-Smith \\ Neurocognitive Development Unit, Institute of Child Health, London, \\ WC1N 1EH, United Kingdom. \\ j.vanherwegen@ich.ucl.ac.uk a.karmiloff-smith@ich.ucl.ac.uk
}

Abstract: The target article by Locke \& Bogin (L\&B) focuses on the evolution of language as a communicative tool. They neglect, however, that from infancy onwards humans have the ability to go beyond successful behaviour and to reflect upon language (and other domains of knowledge) as a problem space in its own right. This ability is not found in other species and may well be what makes humans unique.

The target article by Locke \& Bogin (L\&B) doesn't merely cover language evolution but also takes the whole of human ontogeny into account. However impressive the authors' life history model is, the question remains as to whether it is really language that makes humans intelligent. Throughout the article the authors focus on the development of language as a communicative tool which came into existence through selection, in order to secure the survival of the human species. However, there is no denying that many animals have complex communication systems, elaborate sensorimotor skills, as well as rich mental representations (Premack 2004). Something else, then, must make humans special. We argue that this is the ability in humans to re-represent their representations into an explicit format, transportable from one domain of knowledge to another one. Humans do not only master the basics of the language system for communication, they also reflect upon language as a domain of knowledge. Therefore, language is not merely a communicative tool, it is also a problem space in its own right, making young children behave like little linguists (Karmiloff-Smith 1992).

Even infants are sensitive to the subtleties of the linguistic sound system (Jusczyk \& Aslin 1995). By as early as nine months, for instance, infants have begun to determine the phonotactic sequences of their native language that helps them to segment fluent speech into words (Jusczyk 1999). And, even when they become fluent speakers by about 3 to 4 years of age, children do not just learn the mapping between words and their corresponding referents, but also analyse the morphological system. An example comes from Karmiloff-Smith (1992) when she quotes a passage between a 4-year-old and her mother:

Child: "What's that?"

Mother: "A typewriter."

Child: "No, you're the typewriter, that's a typewrite."

(Karmiloff-Smith 1992, p. 31)

At the age of 4 , the child is not merely focused on communication but on how the system works, that is, that the suffix "er" is agentive and that it is used after verb stems to refer to human agents. This and numerous similar examples show how humans, even young children, consider language as a form of knowledge beyond its use as a communicative tool.

Such explicit consideration of knowledge by children is not only found in the domain of language. Physics is another domain that becomes a problem space in its own right for children. For instance, 5-year-old children are successful at balancing blocks where weight is both evenly and unevenly distributed. However, 7 -year-olds fail to succeed in balancing the unevenly distributed weight blocks. A finer analysis shows that this failure can be explained by their going beyond successful behaviour and creating the geometric-centre theory, believing that all blocks balance at their centre. They ignore counterexamples to their theory, while consolidating it (Karmiloff-Smith \& Inhelder 1975). Only by age 9 years do they accomplish the task at hand when they understand the law of torque. So unlike other species, children do not merely aim for successful behaviour.

If language is not just a communicative tool but also a problem space in its own right, then we can expect to find populations in which linguistic communication is not impaired, but language as a domain of knowledge is, and that having fluent language is not sufficient to ensure peer acceptance. The neurodevelopmental disorder, Williams syndrome, is such an example. Despite IQs in the 50- to 60-point range, individuals with Williams syndrome (WS) have surprisingly proficient language skills (Donnai \& Karmiloff-Smith 2000). Yet, their fluent language does not suffice to get them accepted by peers. In contrast to other adolescents with neurodevelopmental disorders, their peer interaction problems are not caused by reluctance to join in social conversations. So, fluent language and a desire for interaction do not suffice to get a WS adolescent accepted by peers. Outside the field of language, children with WS also do not organize or make their knowledge more explicit. For example, adolescents and adults with WS can learn a vast list of facts; however, they fail to organise these facts into core and peripheral knowledge - a reorganisation process found in normal 9- to 10-year-olds (Johnson \& Carey 1998).

Another population in which the ability to re-represent knowledge is likely to be impaired is Down syndrome, a developmental disorder which, in contrast to WS, involves poor language skills. A longitudinal case study of block balancing involving a Down syndrome child showed that, in contrast to typical developing children, there was no sign over time (from age 9 to age 11 years) of his developing geometric centre theory. Instead, for 19 months the child remained on the level of repeated successful behaviour of the typical 5-year-old. This points to an impairment in the reorganization of knowledge (Karmiloff-Smith 2006).

So even though human language is an impressive communicative tool, humans may be the only species that can re-represent their acquired knowledge through representational redescription. This constitutes an endogenous way of gaining new knowledge, leading to the achievement of creativity and flexible control in humans, in contrast to other species (Karmiloff-Smith 1992). L\&B argue that verbal creativity may have played an important role in the evolution of language, but they don't raise the question as to what makes this verbal creativity possible. We believe that the ability of re-representation, both within and across different domains of knowledge, may turn out to be a possible answer to this question. 